\title{
Chad A. Mirkin Named Outstanding Young Investigator for Work on DNANanoparticle Hybrid Materials
}

Cited for his "pioneering and leadership role in identifying, establishing, and developing a new interdisciplinary field that focuses on using complex biological macromolecules to assemble inorganic nanoparticle building blocks into functional meso- and macroscopic structures," Chad A. Mirkin, the Charles E. and Emma H. Morrison Professor of Chemistry at Northwestern University, is the recipient of the 1999 Outstanding Young Investigator Award from the Materials Research Society. This award recognizes exceptional, interdisciplinary scientific work in materials research by a young scientist or engineer who also displays leadership in the materials area.

In developing a method using DNA linker molecules to assemble nanoparticles into macroscopic aggregates, Mirkin with his research team has rapidly introduced and developed a class of hybrid bioorganic/ inorganic materials and an interdisciplinary field of materials research. His approach to materials synthesis brings together elements of chemistry, biology, materials science, physics, and engineering, opening avenues to a class of nanostructured materials with physical and chemical properties that can be tailored with a high degree of control.

Two years ago, in a seminal paper published in Nature 382 (1996) p. 607, Mirkin described a technique for using the sequence specific interactions of DNA to direct the assembly of particles of different sizes and compositions. Initially, he used this method to show one could "program" the assembly of colloidal gold particles (13-nm diameter), tagged with the appropriate oligonucleotide sequences, into network architectures. These structures exhibited fascinating optical properties that depended upon interparticle distance (controlled by the length of the DNAbased particle linker) and aggregate size.

Mirkin and his co-workers recently showed that binary nanoparticle-based materials also can be prepared via this methodology. In one example, he showed how 31- and 8-nm diameter Au particles, modified with the appropriate oligonucleotides, could be assembled with complementary DNA into periodic network structures. According to his article in the Journal of the American Chemical Society 120 (1998) p. 1959, two general types of structures can be formed. The first is a network structure, with big particle/ small particle periodicity, that canvasses macroscopic areas. The second is a "satellite nanostructure" that consists of one 31-nm diameter particle surrounded by a single layer of 8 -nm particles. He points out that since these are "living

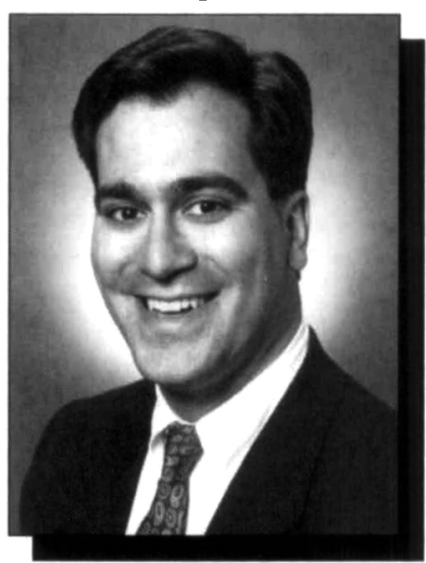

structures" they can be introduced into his assembly scheme to prepare even more sophisticated nanostructured architectures. Therefore, through selection of oligonucleotide and nanoparticle chemical composition and size, Mirkin's method provides control over virtually all physical and chemical properties of the resulting materials.

In 1997, (Science 277, p. 1078), Mirkin and his co-workers demonstrated the first technological application of these novel materials in the development of a highly selective assay for DNA. In this report, Mirkin describes how the colorimetric properties of nanoparticle network materials can be used to differentiate perfectly complementary oligonucleotide targets from strands with single-base imperfections. The assay takes advantage of the intense red color associated with the dispersed nanoparticles and the blue color associated with the network nanoparticle structures. In this test, the target DNA zips together the particles which results in a red to blue color change that can be observed with the naked eye. He and his co-workers have developed a solid-state assay based on these materials that is useful for diagnosing bacterial, viral, and genetic diseases.

In addition to color, these nanostructured materials exhibit unusually sharp melting transitions, which translate into unprecedented sensor selectivity.

Mirkin's research has future commercial possibilities in mass screening for specific nucleotide sequences, screening for biological warfare agents, and a home test for common diseases. This work is also significant since it will allow one to put together nanostructures that will enable one to address many problems in the areas of catalysis, self-assembly, artificial photosynthesis, and multicomponent colloidal crystals.

Mirkin received his $\mathrm{PhD}$ degree in chem- istry in 1989 from The Pennsylvania State University. That same year he moved to the Massachusetts Institute of Technology as a National Science Foundation Postdoctoral Fellow.

Throughout his professional career, Mirkin's research has centered on two main topics: (1) the rational design of hybrid materials for electrochemically controlling transition metal stoichiometric and catalytic reactivity, and (2) surface coordination chemistry and design. Mirkin's contributions have provided an understanding of the important fundamental considerations necessary to control the coordination environments of transition metal centers through appropriate polymeric ligand design and a triggered outer or inner sphere electron transfer event. In addition, he has increased our understanding of the relationship between two-dimensional film structure and function as it pertains to electron transfer and photoreactivity. In the general area of surface design, Mirkin has, in addition to his work with DNA-modified nanoparticles, pioneered the surface modification chemistry of high-temperature superconductors.

Mirkin has received numerous awards for his research, including the American Chemical Society Award in Pure Chemistry, E. Bright Wilson Prize, Phi Lambda Upsilon Fresenius Award, Beckman Young Investigator Award, NSF Young Investigator Award, A.P. Sloan Foundation Fellowship, Office of Naval Research Young Investigator Award, DuPont New Professor Award, and Camille Dreyfus Teacher-Scholar Award. In 1997, he was co-recipient of a prestigious BFGoodrich Collegiate Inventors Award for one of the three most outstanding collegiate inventions in all of medicine, science, and engineering.

Mirkin is the author or co-author of over 90 publications and eight patents and is an active consultant with several major chemical companies. He has given over 120 invited seminars and belongs to various professional organizations, including the Materials Research Society and the American Chemical Society. He is on the Editorial Boards of Advanced Materials, Chemical and Engineering News, and Journal of Cluster Science.

The Outstanding Young Investigator Award will be presented to Mirkin on April 5, 6:00 p.m. at the 1999 MRS Spring Meeting in Salon 7 of the San Francisco Marriott Hotel. He will give his presentation, "DNA-Based Methodology for Preparing Nanocluster Circuits and Arrays" on April 6 at 4:15 p.m. in Symposium W, Golden Gate C2, San Francisco Marriott. 